\title{
Optimization of Learning in General Chemistry II Through the Implementation of the PAIKEM Model to Increase Students' Motivation
}

\author{
Emilia Candrawati ${ }^{1, *}$, Nurlia Latipah $^{2}$, Mellyta Uliyandari $^{1}$

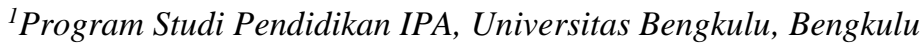 \\ ${ }^{2}$ Program Studi Tadris IPA, IAIN Bengkulu, Bengkulu \\ "Corresponding author. Email: emiliacandrawati@unib.ac.id
}

\begin{abstract}
This study aims to determine the increase in student motivation after applying the PAIKEM (Creative, Effective, and Fun Innovative Active Learning) model to the General Chemistry II course in the S1 Science Education study program, Bengkulu University. This study used a quasi-experimental method with a One Group Posttest Design research design. The research subjects were 27 Semester II class B students of the S1 Science Education Study Program, Bengkulu University. The data was collected using the question instrument at the end of the lesson (posttest) and student response questionnaires. The data were analyzed by Rasch model and SPSS for Likert scale. Based on Rasch Model, the result showed that the mayority of students agree with the implementation of PAIKEM in General Chemistry II courses. Based on SPSS analysis, the results showed that student motivation increased by 48.15 percent for the very high category, 44.44 percent for the high category, and 7.41 percent for the moderate category. Increasing student motivation has an impact on improving student learning outcomes. The class average score for the posttest was 96.7 percent. This value is above the Minimum Completeness Criteria, namely 75. Thus, the PAIKEM model is highly recommended to be applied to General Chemistry II courses.
\end{abstract}

Keywords: PAIKEM Model, student motivation, learning outcomes.

\section{INTRODUCTION}

Education is a learning process for students to have an understanding of something and make it a critical human being in thinking. Education has a goal to educate and develop potential in students. With the growth of intelligence and self-potential, every child can have knowledge, creativity, be physically and mentally healthy, have a good personality, be independent, and become a responsible member of society. Education functions to develop abilities, shape character, and personality so that students become dignified individuals.

Educational innovation should be done to improve the quality of education for the better. Therefore it is necessary to optimize learning by applying the appropriate model. Selection of the right learning model can help lecturers in delivering learning material so that it is easily understood by students. The learning model is a conceptual framework that describes a systematic procedure for organizing learning experiences to achieve certain goals [1].

Based on pre-research observations made by researchers, teacher-centered learning still dominates. This has an impact on the lack of exploration of student abilities and low motivation of students in participating in learning. Motivation has a great influence on the success of the learning process. A person will be successful in learning if he has a desire to learn. For that, it is necessary to apply a learning model that is more creative and innovative so that it can increase student motivation in learning the material [2].

One learning approach that can increase student learning motivation is PAIKEM (Creative, Effective, and Fun Innovative Active Learning). PAIKEM is a teaching approach that is used with certain methods and various teaching media accompanied by a good environmental arrangement so that the learning process becomes active, innovative, creative, effective, and fun [3]. The PAIKEM learning model has many strategies that can be applied by lecturers. One of them is the Everyone is a Teacher Here learning strategy. Everyone is a teacher here strategy is a learning strategy that allows students to be more active and be able to become teachers or mentors for their friends [4]. Through this strategy, students will feel more confident because students are asked to answer questions from friends in their language. Thus, this strategy will be able to increase the inner motivation of students. 


\section{RESEARCH METHOD}

The method used in this study is a quasiexperimental design with One Group Pretest-Posttest Design. Quasi-experiment (pseudo-experiment) is a study that provides treatment and measures the consequences of treatment but does not use a random sample to conclude the changes caused by this treatment [5]. The research was conducted in the Science Education Study Program, University of Bengkulu. Researcher used purposive sampling to determine the sample to be studied and given treatment. Purposive sampling technique is sampling based on certain considerations such as population characteristics or previously known characteristics [6]. The study participants consisted of 27 students.

The instruments used were questionnaires and a test of learning outcomes. A questionnaire with 17 question items was used to measure student motivation after the application of the PAIKEM Learning Model with a learning strategy of everyone is a teacher here. The evaluation test is used to measure student learning outcomes which will be compared with the agreed Minimum Completion Criteria (KKM), namely 75. The questionnaire data uses a Likert scale with the maximum score of the questionnaire item is 4 and the minimum is 1. Data on the response to this questionnaire is analyzed by Rasch model with the Winstep program.

\section{RESULTS AND DISCUSSION}

The learning strategy aims to optimize learning which refers to a general view of an action to determine the method to be used. The Everyone Is A Teacher Here learning strategy is a strategy that can increase student self-confidence and train students to express their opinions on something. With this strategy, students explain the answers as appropriate as an educator or lecturer in the class [7]. This strategy encourages the creation of a comfortable and pleasant classroom atmosphere so that students do not feel awkward expressing their ideas, ideas, and opinions [8]. Appreciation from other students for students who provide explanations in the form of words and actions such as applause can motivate students to dare to express their ideas.

Based on the research results, almost all respondents have a high level of ability in answering the questionnaire items given. In Figure 1. The Maps Person Variable Test, the respondent who has the highest level of ability with codes number 16 and 17 has a logit average of $(+6.45$ logit $)$. From the person variable map data, it can be seen that respondents who have the highest score of ability to answer questions and have the highest ability are 20 respondents, and for respondents who have the lowest ability are respondents numbers 04 and 01 . From the variable map data, it is also seen that even though the respondents are number 04 and 01 have the lowest ability but are still able to answer the most difficult response items, namely number 13 (P13). This means that almost all respondents / students agree to all the questionnaire items given to students, and show that the questionnaire data is valid and reliable.

In the questionnaire items that have the highest level of ability to be answered by the respondent is number 13 (P13 is the questionnaire item number 1) with an average logit item of 2.20. On the map on the right there are 17 questions that have validity levels of varying difficulty starting from P13 which is the most difficult questionnaire item to P9 which has the lowest response item. From the questionnaire data, the question with the lowest difficulty level is question number 9 (P9). This is said to be good because every question given can provide information about the ability of the tested respondent. The M-S-T distance shown on the Wright map above shows that the ability of the respondents is only slightly wider than the distribution found in the difficulty level of the response items. In the context of the level of difficulty contained in the response items, this means that the question items have not too much diversity and the ability of the respondents is not much different. If there is a wider distribution of person abilities than the distribution of items, it can be concluded that the level of person (respondent) ability varies. When comparing the logit item average with the logit person, it can be seen that the logit person is bigger $(+2.20$ logit). This shows that the overall ability is only slightly higher than the difficulty of the question [9]. Analysis on the comparison of the logit person and logit item/ response items based on the map variable map shows that the person logit is much higher than the response item logit. This shows that the overall ability of the respondents is higher than the difficulty level of the response items. This is indicated by almost all respondents agreeing to all aspects of the questionnaire items given.

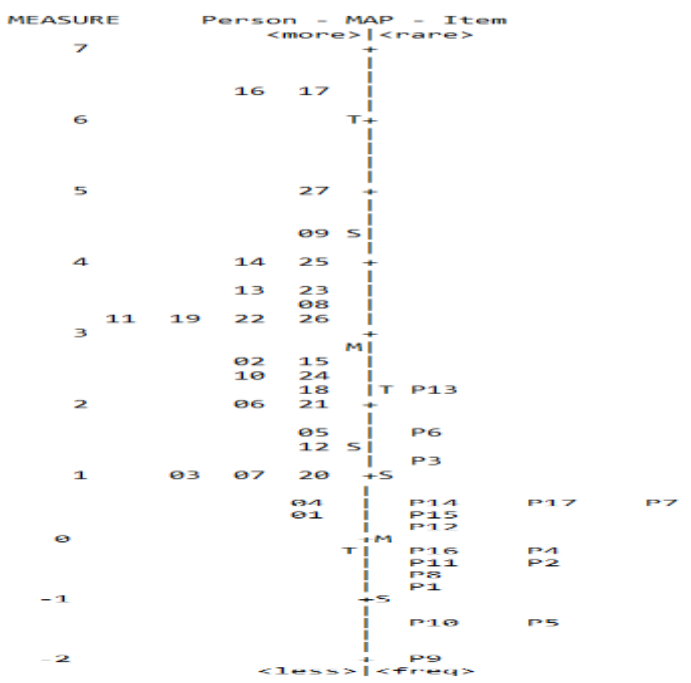

Figure 1. Maps Variable Test (Person)

Analysis of Response Points as shown in the table below shows that the total count is all filled with 27 . This shows that all respondents answered all the items 
given. The difficulty level of the questions shown in the measure column states that the most difficult question is P13 and the easiest is P9.

Table 1. Response Item Analysis Person: REAL SEP.: 2.21 REL.: .83 ... Item: REAL SEP.: 2.13 REL.: .82 Item STATISTICS: MEASURE ORDER

\begin{tabular}{|c|c|c|c|c|c|c|c|c|c|c|c|c|c|}
\hline $\begin{array}{l}\text { |ENTRY } \\
\text { | NUMBERR }\end{array}$ & $\begin{array}{l}\text { TOTAL } \\
\text { SCORE }\end{array}$ & $\begin{array}{l}\text { TOTAL } \\
\text { COUNT }\end{array}$ & MEASURE & $\begin{array}{l}\text { MODELI } \\
\text { S.E. }\end{array}$ & $\begin{array}{l}\text { INF } \\
\text { MNSQ }\end{array}$ & $\begin{array}{l}\text { FIT I } \\
\text { ZSTD|M }\end{array}$ & $\begin{array}{l}\text { OUT: } \\
\text { MNSQ }\end{array}$ & $\begin{array}{l}\text { FIT IP } \\
\text { ZSTD|C }\end{array}$ & $\begin{array}{l}\text { PTMEAS } \\
\text { CORR. }\end{array}$ & $\begin{array}{l}\text { UR-ALIE } \\
\text { EXP.| }\end{array}$ & $\begin{array}{l}\text { EXACT } \\
\text { OBS\% }\end{array}$ & $\begin{array}{c}\text { MATCH } \\
\text { EXP\%| }\end{array}$ & \\
\hline 13 & 76 & 27 & 2.20 & .3511 & 1.83 & 2.412 & 2.27 & 3.11 & .32 & .62 & 48.1 & 66.8 & P13 \\
\hline 6 & 81 & 27 & 1. & . $37 \mid 1$ & 1.26 & & 1.36 & 1.1 & .42 & $.60 \mid$ & 66.7 & $69.8 \mid$ & Dr \\
\hline 3 & 84 & 27 & 1.14 & .381 & .43 & -2.31 & & $-2.3 \mid$ & .73 & .59 & 88.9 & 70.31 & P3 \\
\hline 7 & 87 & 27 & .68 & $.40 \mid 2$ & 2.10 & $2.9 \mid 2$ & 2.02 & $2.5 i$ & .44 & $.58 \mid$ & 63.0 & $70.6 \mid$ & P7 \\
\hline 14 & 87 & 27 & .68 & .40| & .60 & $-1.4 \mid$ & .57 & $-1.4 \mid$ & .65 & . $.58 \mid$ & 77.8 & $70.6 \mid$ & P14 \\
\hline 17 & 88 & 27 & .53 & . 40 & .90 & $-.3 \mid$ & .89 & $-.2 \mid$ & .51 & .57| & 74.1 & $70.4 i$ & \\
\hline 15 & 89 & 27 & .37 & .40 & .67 & $-1.2 \mid$ & .61 & -1.2 & .7 & . $57 \mid$ & 85.2 & $70.5 i$ & \\
\hline 12 & 90 & 27 & .20 & $.41 j^{2}$ & 2.17 & $3.2 \mid 1$ & 1.96 & $2.2 i$ & .32 & $.56 \mid$ & 70.4 & 70.41 & P12 \\
\hline 4 & 92 & 27 & -.13 & . 41 . & .67 & $-1.3 \mid$ & .60 & $-1.1 \mid$ & .63 & $.55 i$ & 77.8 & $70.7 \mid$ & P4 \\
\hline 16 & 92 & 27 & -.13 & .41 & .63 & $-1.5 j$ & .55 & $-1.3 \mid$ & .75 & .55 & 77.8 & $70.7 \mid$ & P16 \\
\hline 11 & 93 & 27 & -.31 & $.42 \mid$ & .45 & -2.51 & .39 & $-1.9 \mid$ & .76 & $.55 \mid$ & 88.9 & $71.3 \mid$ & P11 \\
\hline 2 & 94 & 27 & -.48 & $.42 \mid 1$ & 1.00 & .1 & .91 & $-.1 j$ & . 47 & $.54 \mid$ & 63.0 & $71.6 \mid$ & P2 \\
\hline 8 & 95 & 27 & -.66 & أ.43 & .70 & $-1.2 \mid$ & .67 & $-.7 \mid$ & .62 & أ.53 & 81.5 & $72.1 \mid$ & $P 8$ \\
\hline 1 & 96 & 27 & -.85 & . 43 | 1 & 1.19 & $.8 \mid 1$ & 1.00 & .2 & .51 & .52 & 74.1 & 72. & P1 \\
\hline 5 & 99 & 27 & -1.45 & .46 & .61 & $-1.6 \mid$ & .51 & $-.7 \mid$ & .66 & .48 & 85.2 & 75.8 & P5 \\
\hline 10 & 99 & 27 & -1. & .46| & .82 & $-.6 \mid$ & .68 & $-.3 \mid$ & .66 & . $48 \mid$ & 92.6 & $75.8 \mid$ & P10 \\
\hline 9 & 101 & 27 & -1.90 & . & .76 & $-.8 \mid$ & .55 & $-.4 \mid$ & .56 & أ.44. & 85.2 & $78.8 \mid$ & P9 \\
\hline & & & & & & & & & & & & & \\
\hline & 6.4 & & 1.07 & $.03 \mid$ & .53 & $1.7 \mid$ & & $1.5 \mid$ & & & 11.2 & $2.7 \mid$ & \\
\hline
\end{tabular}

The level of suitability of the question (item fit), whose meaning is in accordance with the ideal measurement model is shown in Table 2. Item fit order. In the table below, it can be seen that the item fit indicators for all question items, namely outfit means square $(0.5<\mathrm{MNSQ}>1.5)$, outfit Z-standard (-2.0 $<$ ZSTD $<+2,0)$, and point measure correlation. $(0.4<\mathrm{Pt}$ Measure corr $<0.85)$, it does not indicate any problem. In other words, all the questions given can be understood well by all respondents, namely students, and there are no questions that are misconceptions [10].

Table 2. Misfit Order

Item STATISTICS: MISFIT ORDER

\begin{tabular}{|c|c|c|c|c|c|c|c|c|c|}
\hline \multirow{2}{*}{\begin{tabular}{|l} 
|ENTRY \\
|NUMBER
\end{tabular}} & \multirow{2}{*}{$\begin{array}{l}\text { TOTAL } \\
\text { SCORE }\end{array}$} & \multirow{2}{*}{$\begin{array}{l}\text { TOTAL } \\
\text { COUNT }\end{array}$} & \multirow[b]{2}{*}{ MEASURE } & \multicolumn{6}{|c|}{ MODELI INFIT | OUTFIT |PTMEASUR-AL|EXACT MATCH| } \\
\hline & & & & S.E. IMNSQ & ZSTD|MNSQ & ZSTD|CORR. & EXP.| OBS\% & EXPon & Item \\
\hline 13 & 76 & 27 & 2.20 & $.35 \mid 1.83$ & $2.4 \mid 2.27$ & $3.1 \mid A .32$ & .62| 48.1 & $66.8 \mid$ & $\mathrm{P} 13$ \\
\hline 12 & 90 & 27 & .20 & $.41 \mid 2.17$ & $3.2 \mid 1.96$ & $2.2 \mid \mathrm{B} .32$ & $.56 \mid 70.4$ & 70.4 & P12 \\
\hline 7 & 87 & 27 & .68 & $.40 \mid 2.10$ & $2.9 \mid 2.02$ & $2.5 \mid \mathrm{C} .44$ & . $58 \mid 63.0$ & $70.6 \mid$ & P7 \\
\hline 6 & 81 & 27 & 1.56 & $.37 \mid 1.26$ & $.9 \mid 1.36$ & $1.1 \mid 0.42$ & .60| 66.7 & $69.8 \mid$ & P6 \\
\hline 1 & 96 & 27 & -.85 & $.43 \mid 1.19$ & $.8 \mid 1.00$ & $.2 \mid \mathrm{E} .51$ & .52| 74.1 & $72.8 \mid$ & P1 \\
\hline 2 & 94 & 27 & -.48 & $.42 \mid 1.00$ & $.1 \mid .91$ & $-.1 \mid F .47$ & .54| 63.0 & 71.6| & P2 \\
\hline 17 & 88 & 27 & .53 & $.40 \mid .90$ & $-.3 \mid .89$ & $-.2 \mid G .51$ & . $57 \mid 74.1$ & 70.41 & P17 \\
\hline 10 & 99 & 27 & -1.45 & $.46 \mid .82$ & $-.6 \mid .68$ & $-.3 \mid \mathrm{H} .66$ & .48| 92.6 & $75.8 \mid$ & P10 \\
\hline 9 & 101 & 27 & -1.90 & $.49 \mid .76$ & $-.8 \mid .55$ & $-.4 \mid \mathrm{I} .56$ & $.44 \mid 85.2$ & $78.8 \mid$ & P9 \\
\hline 8 & 95 & 27 & -.66 & .43|.70 & $-1.2 \mid .67$ & $-.7 \mid \mathrm{h} .62$ & $.53 \mid 81.5$ & $72.1 \mid$ & P8 \\
\hline 4 & 92 & 27 & -.13 & .41 .67 & $-1.3 \mid .60$ & $-1.1 \mid \mathrm{g} .63$ & | $.55 \mid 77.8$ & $70.7 \mid$ & P4 \\
\hline 15 & 89 & 27 & .37 & $.40 \mid .67$ & $-1.2 \mid .61$ & $-1.2 \mid f .76$ & $.57 \mid 85.2$ & $70.5 \mid$ & P15 \\
\hline 16 & 92 & 27 & -.13 & .41 .63 & $-1.5 \mid .55$ & $-1.3 \mid \mathrm{e} .75$ & | 77.8 | 75. & $70.7 \mid$ & P16 \\
\hline 5 & 99 & 27 & -1.45 & $.46 \mid .61$ & $-1.6 \mid .51$ & $-.7 \mid \mathrm{d} .66$ & | $48 \mid 85.2$ & $75.8 \mid$ & P5 \\
\hline 14 & 87 & 27 & .68 & $.40 \mid .60$ & $-1.4 \mid .57$ & $-1.4 \mid c .65$ & | 77.8 & $70.6 \mid$ & P14 \\
\hline 11 & 93 & 27 & -.31 & $.42 \mid .45$ & $-2.5 \mid .39$ & $-1.9 \mid b \quad .76$ & .55| 88.9 & $71.3 \mid$ & | P11 \\
\hline 3 & 84 & 27 & 1.14 & $.38 \mid .43$ & $-2.3 \mid .40$ & $-2.3 \mid \mathrm{a} .73$ & |.59| 88.9 & $70.3 \mid$ & P3 \\
\hline PIEAl & 90.8 & 27.0 & .90 & .41 .99 & $-.3 \mid .94$ & -.1 & 76.5 & 71.7| & \\
\hline P.SD & 6.4 & .0 & 1.07 & .03|.53 & $1.7 \mid .58$ & $1.5 \mid$ & | 11.2 & $2.7 \mid$ & \\
\hline
\end{tabular}

Apart from the level of suitability of the questions, Table 2 also describes the suitability of the items. From the data above, it can be seen that all items for the Outfit Mean Square value, item number 13 have an MNSQ of 2.27 , for ZSTD of 3.1 and a Corr value of 0.32 . All of these values exceed the predetermined criteria. If all the values obtained are not in the specified value range, then this item needs to be reviewed again.

The ZSTD value is influenced by the sample size. If using a very large sample, the ZSTD value will always be above the value 3 . The following shows the suitability curve for response item number 13 where 3 responses are outside the limits of the Outfit confidence space.

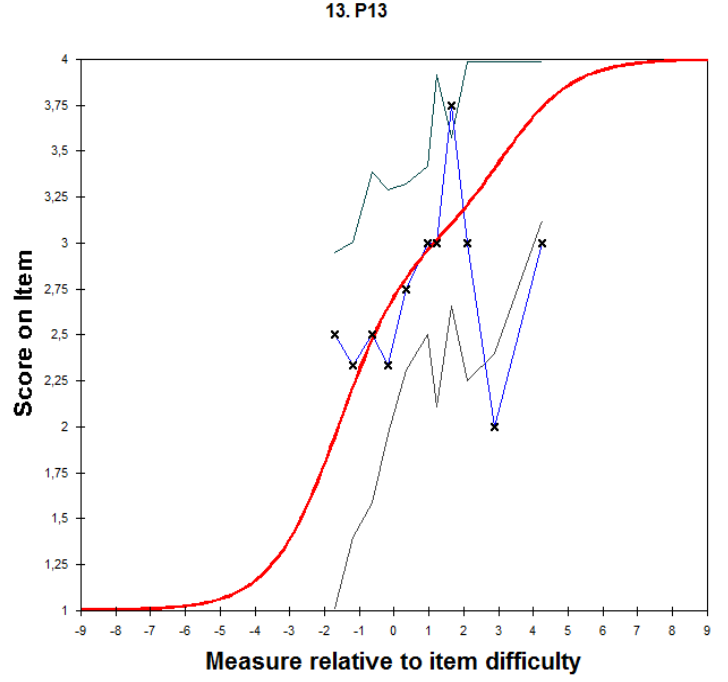

Figure 2. Expected Score ICC

The analysis of the ability of the respondents who filled out the questionnaire items is presented in table 3 . Ability is presented in order from highest to lowest.

Table 3. Person Measure Order Person STATISTICS: MEASURE ORDER

\begin{tabular}{|c|c|c|c|c|c|c|c|c|c|c|c|c|c|}
\hline 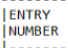 & $\begin{array}{l}\text { TTTAL } \\
\text { SCORE }\end{array}$ & $\begin{array}{l}\text { TOOAL } \\
\text { COUNT }\end{array}$ & MEASURE & $\begin{array}{l}\text { MODEL } \\
\text { S.E. }\end{array}$ & |MNSQ & ZSTD|M & IMNSQ & ZSTD|C & $\begin{array}{l}\text { CORR. } \\
\text {. }\end{array}$ & EXP.। & OBS\% & EXP\%| & Pers \\
\hline 16 & 67 & 17 & 6.45 & 1.06 & \begin{tabular}{|l|l|}
.69 \\
\end{tabular} & - & .21 & $-.4 !$ & .52 & $.24 \mid$ & | 94.1 & $94.1 \mid$ & 16 \\
\hline $\begin{array}{l}17 \\
27\end{array}$ & $\begin{array}{l}67 \\
65\end{array}$ & $\begin{array}{l}17 \\
17\end{array}$ & $\begin{array}{l}6.45 \\
5.98\end{array}$ & $\begin{array}{l}1.06 \mid \\
.68 \mid\end{array}$ & & $\begin{array}{l}-1.1 \mid \\
1.11\end{array}$ & .21 & $\begin{array}{r}-.4 \mid \\
.01\end{array}$ & $\begin{array}{l}.52 \\
.53\end{array}$ & $.24 \mid$ & \begin{tabular}{|l}
94.1 \\
88.2
\end{tabular} & $\begin{array}{l}94.1 \mid \\
83.44\end{array}$ & \\
\hline & $\begin{array}{l}63 \\
63\end{array}$ & 17 & $\begin{array}{l}3.68 \\
4.31\end{array}$ & .58 & & $\begin{array}{l}1.11 \\
\left.3.8\right|_{1}\end{array}$ & 1.96 & 1.71 & $\begin{array}{l}.53 \\
.40\end{array}$ & .42 & \begin{tabular}{|l|}
88.2 \\
88.2
\end{tabular} & $\begin{array}{l}83.4 \mid \\
75.21\end{array}$ & 09 \\
\hline & & & & & & & & & & & & 71.8 & \\
\hline 25 & 62 & 17 & 3.99 & $.55 \mid$ & 1.51 & $\left.1.6\right|_{1}$ & 1.26 & $.7 \mid$ & .41 & .44 & 76.5 & $71.8 \mid$ & 25 \\
\hline 13 & 61 & 17 & 3.70 & .53 & 2.91 & $4.6 \mid 2$ & 2.48 & $3.1 \mid$ & .15 & $.45 \mid$ & $\begin{array}{l}58.8 \\
\end{array}$ & 69.2 & 13 \\
\hline $\begin{array}{r}23 \\
8\end{array}$ & $\begin{array}{l}61 \\
60\end{array}$ & $\begin{array}{l}17 \\
17\end{array}$ & $\begin{array}{l}3.78 \\
3.43\end{array}$ & $\begin{array}{l}.53 \mid \\
.52 \mid\end{array}$ & \begin{tabular}{|l|l|}
.83 \\
71
\end{tabular} & $-.5 \mid$ & & $-.4 \mid$ & .52 & $.45 \mid$ & \begin{tabular}{|l}
82.4 \\
76.5
\end{tabular} & $\begin{array}{l}69.2 \mid \\
67.99\end{array}$ & 23 \\
\hline & $\begin{array}{l}60 \\
59\end{array}$ & $\begin{array}{l}177 \\
17\end{array}$ & $\begin{array}{l}3.43 \\
3.16\end{array}$ & $\begin{array}{l}.521 \\
.51\end{array}$ & & $\begin{array}{r}-1.1 \mid \\
8 \mid 1\end{array}$ & & $\begin{array}{r}-1.1 \mid \\
.8 \mid\end{array}$ & $\begin{array}{l}.63 \\
.64\end{array}$ & .46 & & $\begin{array}{l}67.9 \\
67.2\end{array}$ & \\
\hline 11 & $\begin{array}{l}59 \\
59\end{array}$ & 17 & 3.16 & $\begin{array}{l}.511 \\
.511\end{array}$ & $\begin{array}{l}1.22 \\
1.17\end{array}$ & $\begin{array}{l}.8 \mid 1 \\
.6 \mid 1\end{array}$ & $\begin{array}{l}1.24 \\
1.13 \\
1.13\end{array}$ & $\begin{array}{l}.81 \\
.51\end{array}$ & $\begin{array}{l}.14 \\
.54\end{array}$ & . .46 .46 & \begin{tabular}{|l|}
47.1 \\
58.8
\end{tabular} & $\begin{array}{l}67.21 \\
67.21\end{array}$ & $\begin{array}{l}11 \\
19\end{array}$ \\
\hline 22 & 59 & 17 & & .51 & 1.80 & $\begin{array}{l}.611 \\
\therefore 61\end{array}$ & 1.78 & ..61 & . .50 & .46 & \begin{tabular}{|l|l|}
30.0 \\
82.4
\end{tabular} & 67.21 & 22 \\
\hline 20 & 59 & 17 & 3.16 & .51 & & $3.1 \mid 2$ & 2.22 & 3.01 & .45 & .46 & $\begin{array}{l}52.9 \\
\end{array}$ & 67.2 & 26 \\
\hline & 57 & 17 & $=$ & $.50 \mid$ & & $2.1 \mid 1$ & 1.71 & $1.9 \mid$ & .33 & .46 & 64.7 & $67.4 \mid$ & 02 \\
\hline $\begin{array}{l}15 \\
10 \\
10\end{array}$ & $\begin{array}{l}57 \\
56\end{array}$ & $\begin{array}{l}17 \\
17\end{array}$ & & $\begin{array}{l}.5 \theta \\
.49 \\
\end{array}$ & $\begin{array}{l}\mid 1.00 \\
1.41\end{array}$ & $\begin{array}{r}.1 \mid 1 \\
1.2 \mid 1\end{array}$ & 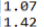 & $.3 \mid$ & $\begin{array}{l}.57 \\
.75\end{array}$ & .46 & \begin{tabular}{|l|l|}
70.6 \\
58.8
\end{tabular} & $67.4 \mid$ & $\begin{array}{l}15 \\
10\end{array}$ \\
\hline $\begin{array}{l}10 \\
24\end{array}$ & $\begin{array}{l}56 \\
56\end{array}$ & $\begin{array}{l}171 \\
17\end{array}$ & & . .49| .49 & & $\begin{array}{l}1.211 \\
-.44\end{array}$ & $\begin{array}{l}1.42 \\
1.83\end{array}$ & $\begin{array}{l}1.21 \\
-.4\end{array}$ & .66 & .46 & $\begin{array}{l}58.8 \\
70.6\end{array}$ & $\begin{array}{l}68.2 \\
68.2\end{array}$ & $\begin{array}{l}10 \\
24\end{array}$ \\
\hline 18 & $\begin{array}{l}30 \\
55\end{array}$ & 17 & $\begin{array}{l}2.41 \\
2.17\end{array}$ & . .49| & 1.1 & $\begin{array}{r}.44 \\
\left..4\right|_{1}\end{array}$ & 1.11 & $\begin{array}{r}. .4 \mid \\
.4 \mid\end{array}$ & $\begin{array}{l}.66 \\
.64\end{array}$ & .46 & \begin{tabular}{|l|}
10.6 \\
58.8
\end{tabular} & $\begin{array}{l}68.21 \\
69.31\end{array}$ & $\begin{array}{l}24 \\
18\end{array}$ \\
\hline 6 & 54 & 17 & 1.93 & .49 & .75 &. .61 & 1.72 & $\therefore 7 \mid$ & .55 & .46 & \begin{tabular}{|l}
30.0 \\
64.7
\end{tabular} & 69.9 & 06 \\
\hline 21 & 54 & 17 & 1.93 & .49 & .30 & $-2.6 \dot{\mid}$ & .28 & $-2.6 \mid$ & .69 & .46 & | 1100.0 & $69.9 \mid$ & 21 \\
\hline & & & & & & $-2.4 \mid$ & & & & $.47 \mid$ & & & \\
\hline 12 & $\begin{array}{l}52 \\
50\end{array}$ & 17 & 1.4 & . & .53 & $-1.3 \mid$ & .53 & -1.31 & .47 & .471 & 82.4 & $\begin{array}{l}70.0 \mid \\
70.9\end{array}$ & 12 \\
\hline & 50 & 17 & & . .47 & .28 & $\begin{array}{l}-1.4 \mid \\
-2.9 \mid\end{array}$ & .47 & $|-3.0|$ & .52 & $\begin{array}{r}.48 \mid \\
.48 \mid\end{array}$ & & 70.9 & 07 \\
\hline 20 & 50 & 17 & 1.01 & .47 & .20 & $-2.9 \mid$ & .17 & -3.0 & .52 & .48 & 100.0 & 70.9 & 20 \\
\hline 4 & 48 & & & .45 & .64 & $-.9 \mid$ & .50 & $-1.3 \mid$ & .32 & .50 & 78.6 & 69.51 & \\
\hline 1 & 47 & 17 & .39 & .44 & | 81 & $-.4 \mid$ & 1.89 & $-.1 \mid$ & .44 & $.51 \mid$ & I 70.6 & 67.81 & \\
\hline & & & & & & & & & & & & & \\
\hline & & & & & & & & & & & & & \\
\hline
\end{tabular}

In Table 3, Person Measure Order, the total count column is the number of questionnaire items answered by the respondent. While the total score column looks at the total score obtained by each respondent. All respondents answered the 17 questionnaire responses given. Respondents who have the highest scores are respondents' number 16 and 17 with measure value $=$ +6.45 . For respondents who have the lowest value with measure $=0.39$, namely respondent number 01 . It can be seen from the data above that several respondents have the same logit value, this means that they show the same raw score (total score), as well as the ability. To 
see further which respondents have higher ability, a scalogram is used. The scalogram can be used to see which respondents have higher ability even though they have the same logit value.

In the aspect of the mismatch of the response with the ideal model as shown by the 6-person fit order table, it can be seen that the least fit is the respondent with codes 13, 09 and 26. This indicates a tendency for an inconsistent pattern among the three respondents in terms of answering these questions.

\section{Table 4. Misfit Person}

\begin{tabular}{|c|c|c|c|c|c|c|c|c|c|c|}
\hline $\begin{array}{l}\mid \text { ENTRY } \\
\text { |NUMBER }\end{array}$ & $\begin{array}{l}\text { TOTAL } \\
\text { SCORE }\end{array}$ & $\begin{array}{l}\text { TOTAL } \\
\text { COUNT }\end{array}$ & MEASURE & $\begin{array}{l}\text { MODEL } \\
\text { S.E. IMNSO }\end{array}$ & $\begin{array}{l}\text { INFFIT } \\
\text { 5e ZSTD } \\
\text { MNST }\end{array}$ & $\begin{array}{l}\text { TFIT |PTMEAS } \\
\text { ZSTD|CORR. }\end{array}$ & $\begin{array}{l}\text { SUR-AL } \\
\text { EXP. }\end{array}$ & $\begin{array}{l}\text { EXACT } \\
1085 \%\end{array}$ & $=$ & | Perso \\
\hline 13 & 61 & 17 & 3.70 & $.53 \mid 2.91$ & $\begin{array}{ll}91 & 4.6 \mid 2.48\end{array}$ & $3.1 \mid \mathrm{A} .15$ & .45 & 58.8 & 69.2 & 13 \\
\hline 9 & 63 & 17 & & \begin{tabular}{l|l|l|}
.58 .70 \\
\end{tabular} & $\begin{array}{ll}70 & 3.8 \mid 1.96\end{array}$ & $1.7 \mid \mathrm{B} .48$ & .42 & 288.2 & 75.2 & \\
\hline $\begin{array}{r}26 \\
2\end{array}$ & 59 & 17 & $\begin{array}{l}3.16 \\
2.66\end{array}$ & $\begin{array}{l}.51 \mid 2.15 \\
5\end{array}$ & $\begin{array}{ll}15 & 3.1 \mid 2.22\end{array}$ & $\begin{array}{l}3 . \theta j c .45 \\
3.9 \mid 8.33\end{array}$ & .46 & 52.9 & 67.2 & 26 \\
\hline${ }_{14}^{2}$ & & & $\begin{array}{l}2.66 \\
3.99\end{array}$ & $\begin{array}{l}.50 \mid 1.76 \\
.55 \backslash 1.53\end{array}$ & $\begin{array}{l}.111 .71 \\
7 \mid 1.48\end{array}$ & $\begin{array}{l}1.9 \mid \mathrm{D} .33 \\
1.0 \mid .36\end{array}$ & .46 & 564.7 & $\mid \begin{array}{r}67.4 \\
71.8\end{array}$ & 02 \\
\hline & $\begin{array}{l}62 \\
62\end{array}$ & $\begin{array}{l}17 \\
17\end{array}$ & $\begin{array}{r}3.99 \\
3.99\end{array}$ & $\begin{array}{l}. .55 \mid 1.53 \\
.55 \mid 1.51\end{array}$ & $\begin{array}{ll}53 & 1.771 .48 \\
51 & 1.6 \mid 1.26\end{array}$ & $\begin{array}{l}1.0 \mid \mathrm{E} .36 \\
.7 \mid \mathrm{F} .41\end{array}$ & .44 & $\begin{array}{l}47.6 \\
476.5 \\
4\end{array}$ & $\left|\begin{array}{l}71.8 \\
71.8\end{array}\right|$ & $\begin{array}{l}14 \\
25\end{array}$ \\
\hline & $\begin{array}{l}62 \\
65 \\
-10\end{array}$ & 17 & 3.99 & $8 \mid 1.45$ & \begin{tabular}{ll|l}
11 & 1.1 .20
\end{tabular} & .016 .53 & .36 & 58.2 & $\mid \begin{array}{l}1.8 \\
83.4\end{array}$ & 23 \\
\hline 10 & 56 & 17 & 2,41 & \begin{tabular}{l|l}
.49 & 1.41
\end{tabular} & \begin{tabular}{ll|l}
41 & 1.2 & 1.42
\end{tabular} & $1.21 \% .75$ & .46 & 58.8 & 68.2 & \\
\hline 11 & 59 & 17 & 3.16 & $.51 \mid 1.22$ & $.8 \mid 1.24$ & $.8 \mid \mathrm{I} .14$ & .46 & \begin{tabular}{l|l}
5 & 47.1
\end{tabular} & 67.2 & 11 \\
\hline $\begin{array}{l}19 \\
18\end{array}$ & $\begin{array}{l}59 \\
55\end{array}$ & 17 & 3.16 & $\begin{array}{l}.51 \\
.51 .17\end{array}$ & . $.6 \mid 1.13$ & $.5[j .54$ & .46 & 58.8 & 67.2 & 19 \\
\hline $\begin{array}{l}18 \\
15\end{array}$ & $\begin{array}{l}55 \\
57\end{array}$ & $\begin{array}{l}17 \\
17\end{array}$ & 2.17 & $\begin{array}{l}.49 .1 .11 \\
.58 \mid 1.80\end{array}$ & $.4 \mid 1.11$ & $\begin{array}{l}.4 \mid K .64 \\
3.57\end{array}$ & .46 & 58 & 69.3 & 18 \\
\hline 1 & $\begin{array}{l}57 \\
47\end{array}$ & $\begin{array}{l}17 \\
17\end{array}$ & $\begin{array}{r}2.66 \\
.39\end{array}$ & $\begin{array}{l}.56 \mid 1.80 \\
.44 \mid .81\end{array}$ & $\begin{array}{l}.11067 \\
\therefore .40 .89\end{array}$ & $\begin{array}{r}.31 \mathrm{~L} .57 \\
-.11 \mathrm{M} .44\end{array}$ & .51 & $\begin{array}{l}57.6 \\
1170.6\end{array}$ & $\mid \begin{array}{l}67.4 \\
67.8\end{array}$ & $\left|\begin{array}{l}15 \\
01\end{array}\right|$ \\
\hline 23 & 61 & 17 & 3.70 & \begin{tabular}{l|l}
.53 & .83 \\
\end{tabular} & \begin{tabular}{l|l|l}
-.5 & .83
\end{tabular} & $\begin{array}{r}-110.44 \\
-.4 \mid \mathrm{N} .52\end{array}$ & .45 & 82.4 & $\mid \begin{array}{l}69.2 \\
\mid 69.2\end{array}$ & 23 \\
\hline 24 & 56 & 17 & 2.41 & \begin{tabular}{l|l}
$.49 \mid .83$ \\
\end{tabular} & \begin{tabular}{l|l}
-.4 & .83
\end{tabular} & $\begin{array}{l}-.41 \mathrm{~m} .66 \\
\end{array}$ & .46 & 5170.6 & 68.2 & 24 \\
\hline 22 & 59 & 17 & 3.16 & .51 .80 & \begin{tabular}{l|l}
-.6 & .78
\end{tabular} & $-.6 \mid 1.50$ & .46 & 52.4 & 67.2 & 22 \\
\hline $\begin{array}{l}6 \\
8\end{array}$ & & 17 & 1.93 & \begin{tabular}{l|l|l}
.49 & .75 \\
5 &
\end{tabular} & \begin{tabular}{ll|l}
75 & -.6 & .72
\end{tabular} & $-.7 \mid k .55$ & .46 & 564.7 & 69.9 & 06 \\
\hline & $\begin{array}{l}68 \\
67\end{array}$ & $\begin{array}{l}17 \\
17\end{array}$ & $\begin{array}{l}3.43 \\
6.45\end{array}$ & \begin{tabular}{r|r}
.52 & .71 \\
1.66 & .69 \\
\end{tabular} & \begin{tabular}{ll|l|l|}
11 & -1.1 & .64 \\
9 & -1 & -71
\end{tabular} & $\begin{array}{r}-1.11 j \\
-4]\end{array}$ & .24 & $\begin{array}{l}56.5 \\
4 \\
4\end{array}$ & $\begin{array}{l}6.9 \\
94.1\end{array}$ & $\begin{array}{l}08 \\
16\end{array}$ \\
\hline 17 & 67 & 17 & $\begin{array}{l}.045 \\
6.45\end{array}$ & \begin{tabular}{l|l}
1.06 & .69 \\
1.06 & .69
\end{tabular} & 59 \begin{tabular}{ll|l}
-.11 & .21 \\
9
\end{tabular} & $\begin{array}{l}-.4 \mid 1.32 \\
-.4 \mid \mathrm{h} .52\end{array}$ & .24 & $\begin{array}{l}4{ }_{1} \\
94.1\end{array}$ & 94.1 & 17 \\
\hline 19 & 48 & 17 & .59 & \begin{tabular}{r|r|}
.45 & .64 \\
.45
\end{tabular} & $\begin{array}{llll}54 & -.99 & .50\end{array}$ & $-1.3 \lg .32$ & .50 & 70.6 & 69.5 & 04 \\
\hline 12 & & 17 & 1.46 & $.48 \mid .53$ & $\begin{array}{lll}53 & -1.31 .53\end{array}$ & $-1,3 \mid f .47$ & .47 & 82.4 & $|70.0|$ & 12 \\
\hline & 50 & 17 & 1.01 & $.47 \mid .49$ & $\begin{array}{lll}19 & -1.4 & .43\end{array}$ & $-1.6 \mid \mathrm{e} .69$ & .48 & 3188.2 & 70.9 & 03 \\
\hline & 53 & & & $.49 \mid .30$ & \begin{tabular}{ll|l}
30 & -2.4 & .27
\end{tabular} & $-2.5 / \mathrm{d} .57$ & & 7| 94.1 & & 95 \\
\hline $21+2+3$ & $\begin{array}{l}54 \\
50 \\
50\end{array}$ & ${ }_{17}^{17}$ & $1:$ & $\begin{array}{r}.49 \mid \\
.47 \mid .30 \\
.20\end{array}$ & \begin{tabular}{ll|l}
30 & -2.6 & .28 \\
20
\end{tabular} & $\begin{array}{l}-2.6 \mathrm{c} c \\
-3.91 \mathrm{~b}\end{array}$ & & $\begin{array}{l}5100 \theta \\
81100 \theta\end{array}$ & $\mid \begin{array}{l}69.9 \\
70.9\end{array}$ & $\begin{array}{l}21 \\
07\end{array}$ \\
\hline 20 & 50 & $\begin{array}{l}17 \\
17\end{array}$ & $\begin{array}{l}1.01 \\
1.61\end{array}$ & .471 .26 & \begin{tabular}{ll|l}
20 & -2.9 & .17
\end{tabular} & $\begin{array}{l}-3.0 \mid \text { a. } \\
\text { - } 52\end{array}$ & .48 & 1) 1100.6 & 70.9 & 20 \\
\hline & 57.1 & 17.0 & & $5 \mid 1.06$ & .1|.94 & $\therefore$ & & 76.5 & & \\
\hline & 4 & & & . $.15 \mid$ & $1.9 \mid .62$ & $1.6 \mid$ & & 15.2 & 1.10 & \\
\hline
\end{tabular}

In Table 4. Misfit Person above, it can be seen that all respondents have at least 1 predetermined limit criteria value. To see a more complete fit, see the following Guttmann matrix.

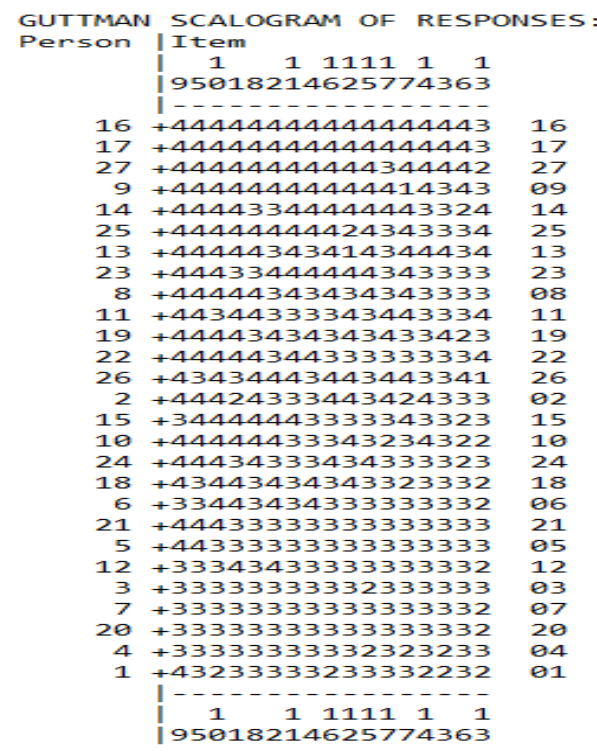

Figure 3. Scologram (Matrix Guttman)

From the picture above, we can see the pattern of answers from each respondent. There are similarities in the answers between 27 respondents who have the highest ability, one of them is from respondents' number 16 and 17, and for respondents' answer patterns with numbers 07 and 20 also have the same answer pattern. The similarity in response patterns could be an indication that the respondents were cooperating with each other or cheating in the implementation of filling out the questionnaire items so that it needs to be reevaluated. It can be seen that the scologram has an irregular / inconsistent response pattern with the answer, this also shows that on certain points he tends to agree with the statement (getting a high / difficult item score) while on other items he tends to reject (get a low item score /easy). This is probably because at that time the respondents lacked the motivation to respond to the scale. This low motivation causes them to give careless responses. The implication is that their responses are varied and inconsistent, depending on their mood when responding to items. However, this does not affect the overall response results [11].

The motivation questionnaire data were also analyzed using SPSS with a Likert scale. The results of the analysis show that 13 students or $48.15 \%$ are in the very high category, 12 students or $44.44 \%$ are in the high category, while the remaining 2 students or $7.41 \%$ are in the sufficient category. Overall, the increase in student motivation is in the high category.

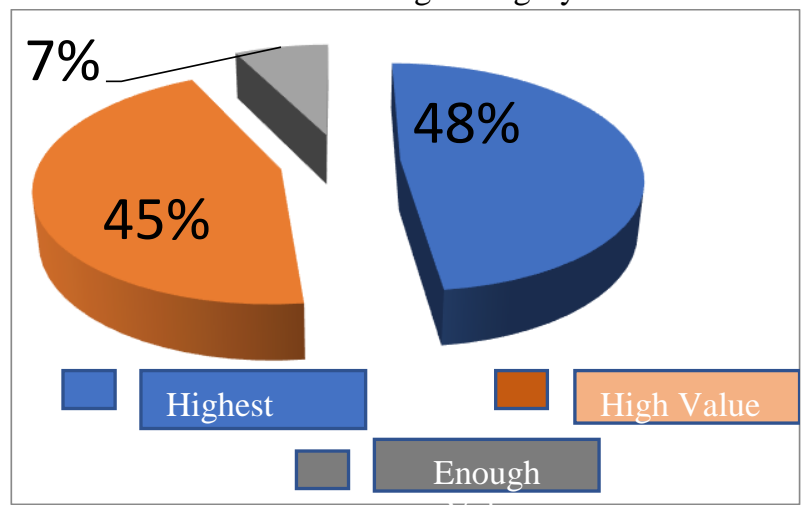

Figure 4. The percentage increase in student motivation

Student posttest as a result of learning showed that all students were able to score above the Minimum Graduation Criteria (KKM), namely 75 . The average posttest score obtained by students was 96.67 .

\section{CONCLUSION}

From the analysis that has been done, it can be coconcluded that the quality of the response questionnaire to the PAIKEM learning model is very good so that it can be used to determine the increase in student motivation after learning with the Everyone is a Teacher Here strategy as one of the PAIKEM models. The results of the analysis of respondents and questionnaire items showed that the majority of students agreed to all the questionnaire items given. This means that the majority of students admit that there is an increase in motivation after participating in learning with PAIKEM. The percentage increase in student motivation, namely $48.15 \%$ is in the very high category, $44.44 \%$ is in the high category, and $7.41 \%$ is in a sufficient category. All students were able to achieve posttest scores above the KKM with an average of 96.67. The PAIKEM model is very well applied to the 
General Chemistry course, but further studies are needed for other courses.

\section{REFERENCES}

[1] S.W. Udin. Model-model Pembelajaran Inovatif. Jakarta: Pusat Antar, 2001.

[2] A.M. Sardiman. Interaksi dan Motivasi Belajar Mengajar. Jakarta: PT. Raja Grafindo Persada, 2007

[3] R. Hartono. Ragam Model Mengajar yang Mudah Diterima Murid. Jogjakarta: DIVA Press, 2014.

[4] M. Silberman. Active Learning 101 Cara Belajar Siswa Aktif. Bandung: Penerbit Nuansa, 2016.

[5] Sugiono, Metodologi Penelitian Pendidikan. Jakarta: Rineka Cipta, 2010.

[6] S. Notoatmodjo. Metodologi Penelitian Kesehatan. Jakarta: Rineka Cipta, 2010.
[7] Hamruni. Strategi Pembelajaran. Yogyakarta: Insan Madani, 2012.

[8] A. Saefuddin. Pembelajaran Efektif. Bandung. PT Remaja Rosdakarya, 2014.

[9] H. Untary, E. Risdianto, and Kusen, Analisis Data Penelitian dengan Model Rasch dan Winstep. Bengkulu: Halaman Moeka, 2020.

[10] B. dan W. Sumintono, Aplikasi Permodelan Rasch pada Assessment Pendidikan. cimahi: Trim Komunikata, 2015.

[11] E. Risdianto, A. Darmawan, M. Kristiawan, Wachidi, and Riyanto, "Rasch model analysis on the feasibility test of basic physics II practical guide using augmented reality," ARPN J. Eng. Appl. Sci., vol. 15, no. 4, pp. 482-490, 2020. 